\title{
Argument Visualization for eParticipation: Towards a Research Agenda and Prototype Tool
}

\author{
Neil Benn and Ann Macintosh \\ Institute of Communications Studies, University of Leeds, Leeds, LS2 9JT, UK \\ \{N.J.L.Benn, A.Macintosh\} @leeds.ac.uk
}

\begin{abstract}
This paper describes research that aims to develop an argument visualization tool and associated method for supporting eParticipation and online deliberation. Based on the state-of-the-art in the field of computer-supported argument visualization, the tool will support the work of relevant eParticipation actors by enabling them to navigate through arguments contained in relevant consultation and policy documents. This tool will form the core of our investigation into the mediating role that large, Web-based argument maps can play in eParticipation scenarios. In particular, we intend to investigate the method and practice of how various eParticipation actors use the tool in the policymaking process. To this end, this paper sets out a clear research agenda for research at the intersection of eParticipation and computer-supported argument visualization.
\end{abstract}

Keywords: Argument visualization, technologies for eParticipation, online deliberation.

\section{Introduction}

This paper describes research and development on an argument visualization tool (AVT) for supporting eParticipation and online deliberation. The AVT is part of a larger suite of tools being developed within the EU-funded IMPACT project. The project began January 1, 2010 and will run for three years. The aims of the IMPACT project include addressing the four overarching problems outlined in [1], namely:

1. How can the various actors determine the relationships between contributions to policy development, whether taken from expert papers, consultations or public forum discourse, and appreciate how these contributions are taken through to decisions?

2. How can the unstructured text from the various information sources be analyzed to enable the reconstruction of formal arguments?

3. How can the actors understand better what critical questions to ask in order to determine the validity of the information put forward?

4. Given the large, dynamic nature of the information base, how can the actors identify which issues are of importance to them and how can they be supported to make reasoned contribution to the policy development? 
IMPACT does this by combining the two distinct types of argumentation tools described by Bex et al., namely knowledge-based tools and sense-making tools [2]. The intention is to integrate tools that contain knowledge about the problem domain and can perform reasoning to suggest solutions to the problem with argumentation tools described as sense-making systems [3] which typically do not support reasoning but rather structure the problem, by using visualization techniques. In addition to the AVT tool, IMPACT will develop and integrate three other argumentation-based tools relating to the following.

- Argument reconstruction: investigating how and to what extent data resources distributed throughout the Internet can be searched and arguments marked up in such a way as to enable them to be semi- automatically aggregated, analyzed and visualized.

- Policy modeling and analysis: using techniques from the field of AI and Law to allow stakeholders to simulate the legal effects of policy proposals.

- Structured consultation: extending prior research on using argumentation schemes to generate focused surveys in order to support argumentation schemes needed for policy deliberations and generate surveys.

Importantly, all the tools will be based on the same computational model of argumentation. Further details of IMPACT and the consortium can be found on the project website $^{1}$.

The AVT is intended to support the work of relevant actors by enabling them to navigate through arguments contained in relevant consultation and policy documents. To adequately achieve this goal, the AVT will be based on the state-of-the-art methods and tools in the field of computer-supported argument visualization (CSAV). However, the rationale for the AVT is grounded firstly in current eParticipation research priorities, which seek technological support for improvements in the efficiency, inclusiveness, openness and accountability of public services and democratic processes.

Thus, in addition to developing the AVT tool and exploring how best to improve the readability of very large visualizations of arguments (often referred to as 'argument maps'), we will investigate the mediating role that such large, Web-based argument maps can play in eParticipation scenarios. In particular, we intend to investigate the method and practice of how relevant eParticipation actors use the AVT tool in the policy-making process. To this end, the aim of this paper is to set out a clear research agenda for our research at the intersection of eParticipation and CSAV.

Specifically, §2 introduces the specific eParticipation and CSAV challenges that motivate our research and drive the development of the AVT. Next, $\$ 3$ describes some preliminary design choices made with respect to our AVT development. Finally, $\S 4$ and $\$ 5$ conclude the paper by describing the set of research questions that will guide our research intersecting eParticipation and CSAV.

${ }^{1}$ IMPACT stands for Integrated Method for Policy making using Argument modelling and Computer assisted Text analysis: http://www.policy-impact.eu 


\section{Drivers of the AVT}

This section describes the main drivers of our AVT development. The rationale for the AVT is grounded firstly in current eParticipation research priorities, which seek technological support for improvements in the efficiency, inclusiveness, openness and accountability of public services and democratic processes (\$2.1). Secondly, the AVT is grounded in current argument visualization research priorities specifically as they relate to needs for improvement in existing visualization tools for policy consultation $(\$ 2.2)$.

\section{1 eParticipation Drivers}

There is a wide body of research that recognizes the belief that the Internet and other digital technologies have the potential to broaden and deepen the democratic process, making it more transparent, inclusive and accessible [4][5]. However, other researchers argue that the capacity of information and communication technologies to facilitate online deliberative engagement on policy issues has not been as significant as was originally believed or hoped for [6]. They suggest:

Demands on knowledge technologies include meeting the need to support rational and justified argumentation, establishing the best balance between a structured format, traceability of contributed information, its accountability in use and transparency about how much information is needed or used to inform policy debate. Additionally, technology design has to consider whether any structuring of information creates boundaries and borders that can limit the access to and understanding of content.

Furthermore, [7] ask the question "How can vast numbers of people engage in collective talk without the voices of individuals being drowned out by the noise of the crowd?" They go on to suggest that a possible radical solution would be for argument visualization research to provide meaningful graphical representations of large-scale discussions, so that the process of accessing and making collective sense of the evolving views of people need not involve reading every word of text produced. The objective of our research and development on the AVT tool is to make possible this 'radical solution'.

\subsection{Argument Visualization Drivers}

There are an increasing number of researchers reflecting on the use of CSAV methods and tools to support what is referred to as "sense-making" - literally, the task of making sense of some complex discourse in order to understand the structure of the discourse and the main moves being made in the discourse. For an overview of this strand of CSAV research see [3] and [8]. Some researchers (e.g. [9][10][11]) are specifically considering the policy-consultation domain and have experimented with various argument visualization tools for addressing the challenges within this domain.

However, applying CSAV tools in this way also presents its own challenges, as was most recently identified during a specially convened one-day invitational workshop which brought together a cluster of researchers to consider the emerging 
argument visualization tools that have the potential to support online deliberation ${ }^{2}$. The specific result of this workshop was a statement of the top ten key adoption challenges of introducing argument visualization tools to support consultative policymaking. These challenges are visualized in Fig. 1.

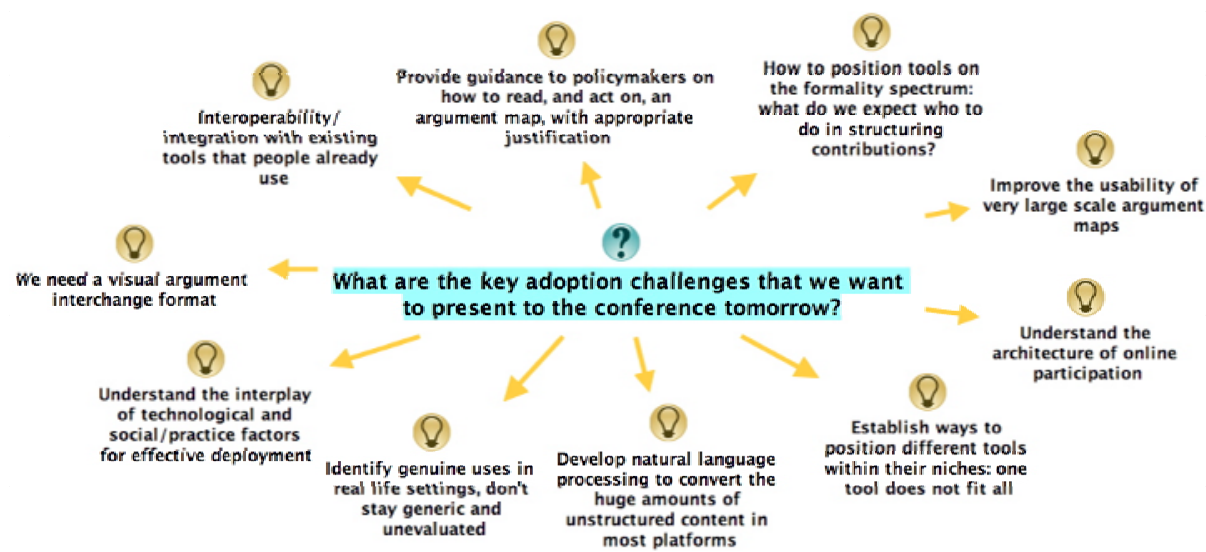

Fig. 1. The top ten adoption challenges faced by argument visualization tools for supporting online deliberation. (Map is courtesy of Simon Buckingham Shum retrieved from http:// cloudworks.ac.uk/cloud/view/3667)

Furthermore, underlying these ten challenges, and a repeated theme throughout the workshop was the readability and usability of visual depictions of argumentation. As [12] indicates, reading argumentative structures, whether in text or in graphical form has always been a difficult skill to acquire. This is because making sense of the argumentative structure requires both having a sense of the detail as well as having a sense of the whole (particularly how one detailed area relates to another detailed area). Exploring these particular argument visualization issues will form a key part our research and development on the AVT.

\section{Designing the AVT}

The objective of the AVT tool is to help users to understand and to make sense of the online policy debate over time, and help the policy analyst to report on the online debate at the end of the consultation period. This section describes how the AVT is designed to meet these objectives. First, we describe the principle of "Documentcentricity" which the AVT tool will adopt (\$3.1). Second, we describe an early design decision, namely to build on the substantial advances made in CSAV technology (§3.2). In particular, we have decided to reuse the Cohere tool [13] as a platform for our AVT development (\$3.3).

${ }^{2}$ http://olnet.org/odet2010 


\subsection{Document-Centricity: Anchoring Online Deliberation in Public Documents}

The main eParticipation usage scenario envisaged by the IMPACT project involves an organization (typically a government agency) publishing a policy-consultation document (a Green Paper in the case of a government agency) in order to solicit feedback from relevant stakeholders. Thus, the visualization generated by the AVT, i.e. the argument map, is anchored in this policy-consultation document, and all arguments generated by stakeholders are entered into the argument map with links to the original policy-consultation document.

In this way all visualized data in the AVT tool will have a connection to the original consultation document. This document-centricity is important since the policyconsultation document is central to our underlying objectives of achieving transparency and understanding in the argument map. Furthermore, this document-centricity promotes sense-making for users joining at any time during a lengthy consultation period as they can see how their arguments fit within the ongoing policy-deliberation process consultation. Finally, this document-centricity gives confidence to the policy-makers that the contributions provided by stakeholders are on-topic and relevant.

From a technological perspective, in order to support this document-centricity, nodes in the Web-based argument map should contain hyperlinks directly to relevant sections and paragraphs in the original policy-consultation document. This presents a challenge since the process of drafting policy-initiatives within organizations in general is typically performed with standard word-processing software and formats such as PDF are used to exchange documents. Such formats do not easily facilitate online hyperlinks to sections of a document

Specifically meeting this challenge of making policy documents citable down to the level of sections and paragraphs is beyond the scope of our AVT development for IMPACT. However, where appropriate, the AVT tool can make use of results from other areas of research and development that have taken on this challenge. For example, some Open Data enthusiasts have recently embarked on a project called Citability.org ${ }^{3}$, which supports making government documents and data available online such that they can be easily referenced for public debate, commentary and analysis. The founders of the Citability.org initiative believe that having the ability to refer to original source documents, down to the level of sections and paragraphs, makes it more difficult to intentionally misrepresent facts and arguments in a debate.

\subsection{State of the Art}

In order to determine the most appropriate starting platform for the IMPACT AVT tool and to ensure it is based on the state-of-the-art in the field of argument visualization a selection of argumentation visualization systems were reviewed. These were:

- Araucaria and OVA $[14,15]$

- Argunet [16]

- Carneades [17]

${ }^{3}$ http://citability.org/ 
- Cohere [13]

- Compendium [18]

- Cope It! [19]

- Debategraph [20]

- Deliberatorium [21]

- LASAD [22]

- Rationale and bCisive [23]

The reviews were based on the literature about the tools and personal communication with the relevant tool developers. The reviews focused on consideration of the drivers for the AVT tool as described previously and the specific requirements of the IMPACT project.

Araucaria and OVA. Araucaria is an argument-diagramming tool developed by the Argumentation Research Group (ARG) at the University of Dundee, UK. It provides a limited analysis of arguments where the user is supported in reconstructing and diagramming an argument. The software supports several different diagramming methods. For example, the latest version of the tool supports Wigmore diagrams, a technique of presenting legal arguments in a diagrammatic form. Whereas Araucaria is a desktop application, OVA (Online Visualization of Argument) is accessible from a web browser, but otherwise is similar to Araucaria in its support for analyzing and mapping arguments. The web-based access allows for built-in support for analysis of web pages by providing a URL.

Argunet. Argunet is a tool for collaborative argument analysis and reconstruction of complex debates. Argunet consists of two software components: an Argument Editor, with which debates can be reconstructed in varying degrees of detail, and an Argument Navigator, a Web-browser-oriented presentation tool with which the public can browse debates. Argunet adopts an argument mapping approach that is based on classical argument structure. That is, arguments are reconstructed as premise- conclusion structures and visually linked to each other with green and red arrows indicating support and attack relationships between arguments, mapped as a directed graph according to their dialectical relations. Complex argumentations are visualized as color-coded maps in which green and red arrows indicate support and attack relations between arguments. Note, however, that in Argunet debates are not the same as argument maps. Rather, since debates might be too complex to be represented in a single argument map, a debate in Argunet may contain any number of argument maps.

Carneades. Carneades is an open-source argumentation system developed during the European Estrella project (IST-2004-027655), which aims to help both citizens and government officials take part more effectively in dialogues for assessing claims, for example claims for social services such as housing or unemployment benefits. Carneades supports a range of argumentation tasks. Specifically, the tool provides software components for constructing arguments from formal models of legal concepts, rules and cases, for evaluating and comparing arguments, applying proof standards and 
respecting the allocation of the burden of proof and argument visualization. One of the strengths of Carneades lies in its ability to inform users about the acceptability of statements without requiring the user to have an expertise in argumentation theory, mathematics or computer science.

Cohere. Cohere is a Web 2.0 system using the familiar standard Issue-Based Information System (IBIS) framework to provide argument analysis and visualization. In the IBIS approach, solving difficult problems involves deliberation. The deliberation process starts with a root issue (expressed as a question), and ideas are offered in response to this issue. Arguments are then brought in that support or object to a particular idea. The elements (i.e. the Issues, Ideas, and Arguments) produced at each stage of the process are then recorded so as to capture the design rationale behind a particular solution to the problem. One of the main features of Cohere with respect to argument visualization is that it provides a platform for collaborative deliberation and mapping of public policy debates over the Internet. In addition, one of the key principles behind Cohere is that any content on the Internet can serve as a node of information in the argument map, any node can be related to any other node and users can specify the properties of the nodes and relationships.

Compendium. Compendium is a hypermedia concept/argument-mapping tool that has been applied in a number of domains including policy deliberation, real-time dialogue mapping of meetings, and scholarly information management. Compendium aims to provide an open mapping environment in the paradigm of the IBIS and argument-based design-rationale approaches. Thus Compendium comes pre-loaded with node and link types derived from IBIS. The system allows for considerable customization of the argument maps by the users and supports outputs in multiple document formats. Elements of a discussion are represented as 'queries' and 'responses', to which qualifying remarks can be attached indicating 'support for', or 'criticism of' that contention. Using hyperlinks, users can associate relevant documents with particular nodes to back-up any references. It is also possible to partition the discussion into a series of linked maps, which has the advantage of breaking down large amounts of data into manageable portions. Finally, users can perform searches upon the information contained in the nodes, which facilitates the extraction of information contained in the maps.

Cope It!. Cope It! is a web-based eParticipation platform designed to support community deliberation, allowing for distributed, synchronous or asynchronous collaboration over the Web. It supports this collaboration through the use of argument mapping and an integrated threaded discussion forum. Argument mapping in Cope It! is based on the IBIS approach. Users can upload various types of "knowledge items" to a collaborative workspace, and these items can be of type Idea, Note, or Comment, or any external multimedia resource that is located on the user's PC or on the Web. Items can be linked and users can choose the color of the link and provide a label describing the intended relationship. Furthermore, Cope It! allows users to cluster related items 
into colored rectangular regions in their workspace. It also provides the potential to 'evaluate' informally the strengths of the arguments through a user voting system.

Debategraph. Debategraph is a web-based application that allows users to enter unstructured arguments, for and against a debate, into the evolving argument map. As such it provides an online, public, multi-user forum to develop and present debates. It is called a wiki debate visualization tool in the sense that users can modify the debate maps in the same way that they might be allowed to collaboratively modify a wiki (although most wikis are text based). Furthermore, each node in the map can be regarded as a mini-wiki that can contain textual as well as multimedia content. Thus, arguments in debate maps are continuously open to challenge and improvement by all users. Debates can be started, modified, and reused by the users. The objective is that over time, the debates become definitive so that Debategraph becomes a public library of very well articulated debate graphs on a range of topics. Elements in Debategraph are derived from the IBIS approach. The core elements are Issues, Positions (i.e. responses to Issues), Supportive Arguments, and Opposing Arguments (which are advanced for and against positions and other arguments). Each element on a map has a comments section to allow for open discussion. Also, each element can be rated in terms of the perceived strength of the point it makes. Finally, part of every map has a direct URL associated with it; so readers can be pointed towards the debate as whole or towards a specific argument within the debate.

Deliberatorium. Deliberatorium (formerly Collaboratorium) is a web-based, collaborative deliberation system that supports large (and likely geographically dispersed) user communities in controversial discussions online. Users can browse and create argument maps (or what the authors also refer to as "deliberation maps"). An argument map consists of linked, user-contributed "posts". Users can contribute new posts, edit existing posts, comment on posts, and rate posts (with the idea that rating will allow the system to highlight the best contributions). Each post is a unique contribution to the system, thus users are prevented from replicating a post that has been made elsewhere in the argument map. Deliberatorium is based on the IBIS approach, thus each post represents a single Issue, Idea, or Pro or Con argument.

LASAD. The LASAD (Learning to Argue: Generalized Support Across Domains) project, funded by the German Research Foundation, is developing a Web-based educational argumentation system. The project is seeking primarily to contribute to the area of Intelligent Tutoring Systems by developing a system specifically aimed at teaching argumentation skills, following in the path of other ITS for teaching argumentation skills such as Belvedere, ARGUNAUT, and LARGO. However, the LASAD project differs from these other research tools in that it aims to produce a generic, flexible, and reusable software architecture, and accompanying methodology, for developing argumentation systems to help students learn argumentation in different domains. As a proof of this concept, the first phase of the LASAD project has demonstrated how this generic software architecture can be used to emulate existing argumentation systems (e.g. Belvedere and LARGO) and existing argumentation 
frameworks (e.g. Toulmin and Walton Argumentation Schemes). The user is able to create a new session from a template (example templates include Belvedere, Carneades, and Toulmin), or join an existing active session. A session loads an argument map, which has an underlying model of argument corresponding to the template used to create the session. Each session can be individually configured to include features such as a chat system, where a user is able to view a list of other online users that s/he can interact with. Note that, through the use of sessions and features such as a chat system, LASAD supports collaborative use as well as individual use.

Rationale and bCisive. Rationale is a desktop-based argument-mapping tool developed by Tim van Gelder at the University of Melbourne and marketed by a company called Austhink. Rationale supports the development of simple diagrams of complex reasoning, so that the evolving argument can be visualized. Primarily, the system is intended for educational use to help students develop their critical thinking skills and develop argumentative essays. However, the tool has been used in more professional settings, such as by lawyers to layout legal arguments and by policy analysts to develop and defend policy. Whereas Rationale is meant to be used by the individual user, bCisive, and specifically the recent version bCisive Online provides a common workspace for real-time collaboration. It is marketed as a tool to support group planning and decision-making, and team problem-solving. Though it is not explicitly described as an argument-mapping tool, the drawing elements derive from the IBIS approach (i.e. users can create Issue, Idea, and Pro and Con nodes), thus the tool can be used to support Dialogue Mapping or Issue Mapping.

Table 1 summarizes the main features of each of these CSAV tools and compares them in terms of how suitable each might be as a platform for the IMPACT AVT tool. Based on the comparison of the tools, Cohere appears to be the most suitable of the existing tools to be used as a platform for the AVT tool.

\subsection{Reusing Cohere as the AVT Platform}

Cohere is an open source, Web2.0 tool for argument analysis and argument visualization. We have decided to use Cohere as a platform for the AVT because it already supports a number of features that we believe the AVT should provide. These features include enabling users to create Web-based argument maps; to add, delete, and edit nodes and relations in an argument map; and to browse and zoom argument maps, making use of hyperlinks embedded in nodes to access further information (e.g. the original source data from which the node is derived).

Furthermore, one of the main principles on which Cohere is built is that when mapping out an argument, users must be able to ground agreement or disagreement in original source documents distributed anywhere on the Web. This document-centric feature of Cohere is important because, as discussed previously, the argument map should be a visualization of arguments anchored in the original policy-consultation document and any other associated documents published during the public consultation process. 


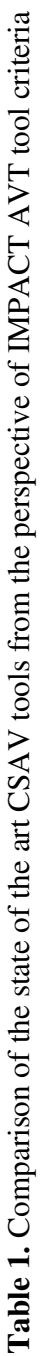

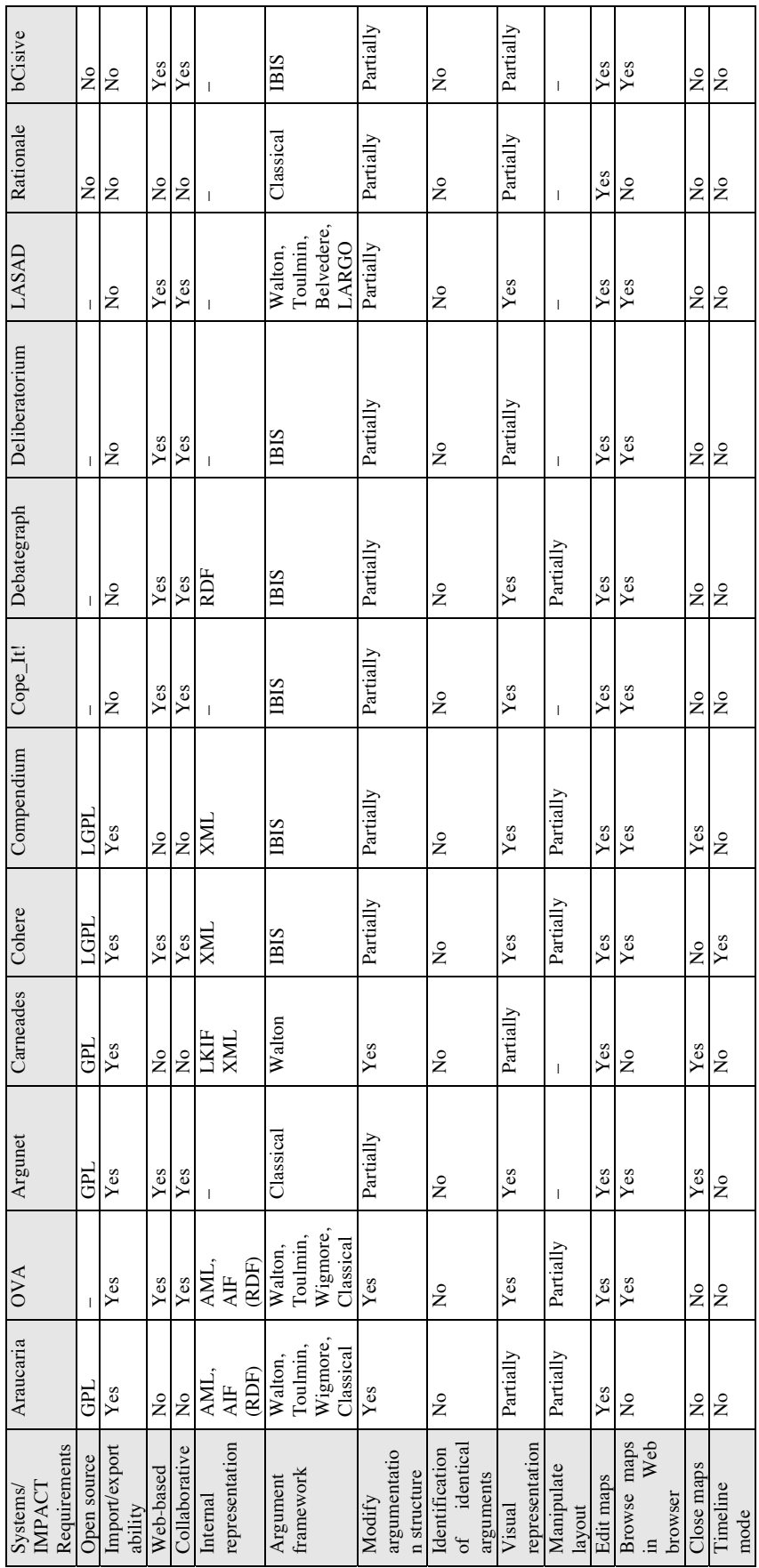


Finally, as an open source tool it can be extended to include the new features as envisaged by our specific IMPACT project usage scenarios. For example, the decision was made within our research project to represent the underlying models of argument in the Legal Knowledge Interchange Format (LKIF) developed during the EU-funded ESTRELLA Project [24]. Using formal semantics, LKIF models can support sense-making tasks such as evaluating arguments to determine which are acceptable. However, the Cohere platform was not originally designed to support LKIF, thus one of the new features it will need to incorporate is a mechanism for input and output of arguments represented in LKIF.

\section{Towards a Research Agenda for Argument Visualization in eParticipation}

Governments are aware that Web-based approaches to facilitate consultation deliberations on policy issues, and provide access to government and expert reports and the documents discussed, may provide advantages for them and those effected by the policy, in terms of better understanding of policy issues, more effective deliberative communication, and more evidence-based decision-making. With regard to the AVT, the new ways the stakeholders will interact, and therefore the initial evaluation criteria (shown in italic) are summarized in Table 2.

In light of these potential new interactions, in our work we aim to investigate how CSAV can foster more substantive understanding and engagement by various eParticipation stakeholders in consultations on complex public issues. This has led us to devise a set of research questions to guide our work:

Q1. How should one design web-based argument maps for policy consultation on a complex issue that allows various stakeholders to browse, navigate and view the ongoing debate?

Output: Methodology for design of interactive Web-based argument visualization tools

Q2. How should one use CSAV techniques to interpret formal models of arguments and what level of granularity is meaningful to the stakeholders.

Output: Method and associated application to interpret models.

Q3. Can we find evidence of the advantages of argument visualization tools for representing the policy-deliberation process?

Output: Evidence based on real-world scenarios of use

Q4. How can we fill the gap in CSAV research dealing with deriving a visual language for argument mapping?

Output: A set of visual principles that describe the essential visual cues and features that are needed to visually depict argumentation to better support sense-making. 


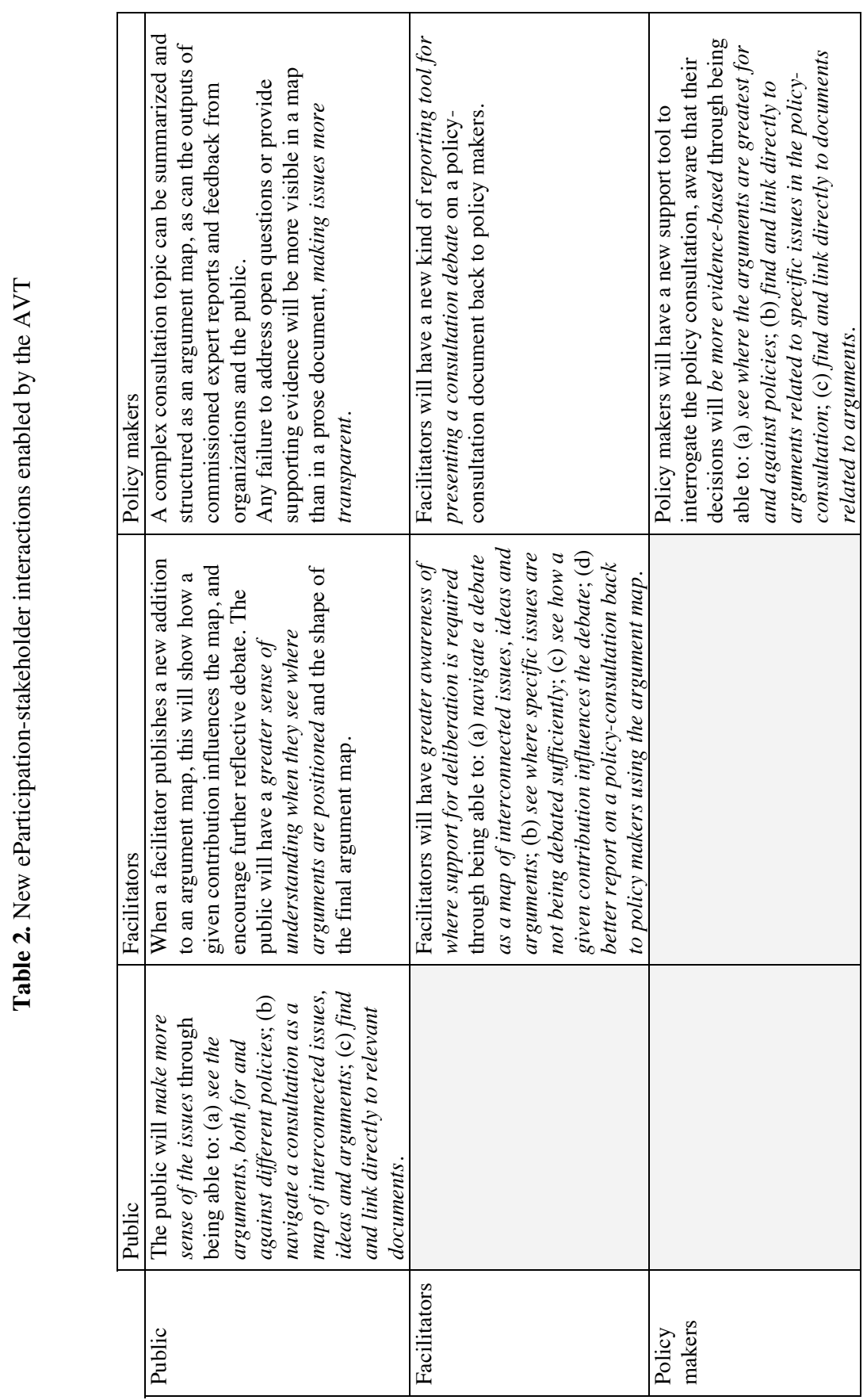




\section{Conclusion}

This paper has introduced our work on developing an argument visualization tool (AVT) for supporting eParticipation and online deliberation. It presented particular challenges that drive the research and development on this AVT. Furthermore, the paper presented some preliminary design decisions for the AVT in order to meet the challenges that were highlighted. Finally, the paper put forward a set of research questions that will guide our future work on this topic which is at the intersection of two vibrant research fields, eParticipation and computer-supported argument visualization.

Acknowledgments. This work is partially funded by the European IMPACT project (IST-FP7-247228) an EU-Commission grant.

\section{References}

1. Macintosh, A.: Moving Towards "Intelligent" Policy Development. IEEE Intelligent Systems 24(5), 79-82 (2009)

2. Bex, F., Prakken, H., Reed, C., Walton, D.: Towards a formal account of reasoning with evidence: Argumentation schemes and generalizations. Artificial Intelligence and Law 11(2-3), 125-165 (2003)

3. Kirschner, P., Buckingham Shum, S., Carr, C.: Visualizing Argumentation: Software Tools for Collaborative and Educational Sense-Making. Springer, London (2003)

4. Blumler, J.G., Gurevitch, M.: The New Media and Our Political Communication Discontents: Democratizing Cyberspace. Information, Communication \& Society 4(1), 1-13 (2001)

5. Dahlgren, P.: The Internet, Public Spheres, and Political Communication: Dispersion and Deliberation. Political Communication 22(2), 147-162 (2005)

6. Macintosh, A., Coleman, S., Schneeberger, A.: eParticipation: The Research Gaps. In: Macintosh, A., Tambouris, E. (eds.) ePart 2009. LNCS, vol. 5694, pp. 1-11. Springer, Heidelberg (2009)

7. Coleman, S., Blumler, J.G.: The Internet and Democratic Citizenship. Cambridge University Press, New York (2009)

8. Okada, A., Buckingham Shum, S., Sherborne, T.: Knowledge Cartography: Software Tools and Mapping Techniques. Springer, Heidelberg (2008)

9. Macintosh, A., Gordon, T.F., Renton, A.: Providing Argument Support for eParticipation. Journal of Information Technology \& Politics 6(1), 43-59 (2009)

10. Snaith, M., Lawrence, J., Reed, C.: Mixed Initiative Argument in Public Deliberation. From e-Participation to Online Deliberation. In: Proceedings of the Fourth International Conference on Online Deliberation, OD 2010, Leeds, UK, pp. 2-13 (2010)

11. De Liddo, A., Buckingham Shum, S.: Capturing and Representing Deliberation in Participatory Planning Practices. In: De Cindio, F., Macintosh, A., Peraboni, C. (eds.) From eParticipation to Online Deliberation, Proceedings of the Fourth International Conference on Online Deliberation (OD 2010), Leeds, UK, pp. 27-40 (2010)

12. Kolb, D.: The Revenge of the Page. In: Proceedings of the 21st ACM Conference on Hypertext and Hypermedia (HT 2008), pp. 89-96. ACM, Pittsburgh (2008) 
13. Buckingham Shum, S.: Cohere: Towards Web 2.0 Argumentation. In: 2nd International Conference on Computational Models of Argument (COMMA 2008), pp. 97-108. IOS Press, Amsterdam (2008)

14. Reed, C., Rowe, G.: Araucaria: software for argument analysis, diagramming and representation. International Journal on Artificial Intelligence Tools 13, 961-980 (2004)

15. Snaith, M., Lawrence, J., Reed, C.: Mixed Initiative Argument in Public Deliberation. In: From e-Participation to Online Deliberation, Proceedings of the Fourth International Conference on Online Deliberation, OD 2010, Leeds, UK, pp. 2-13 (2010)

16. Betz, G., Bohse, H., Voight, C.: Perspectives for Argunet in eParticipation. Paper presented at the DEMO-net Workshop on Argumentation Support Systems for eParticipation, Berlin, Germany (March 5, 2007)

17. Gordon, T.F.: An Overview of the Carneades Argumentation Support System. In: Tindale, C.W., Reed, C. (eds.) Dialectics, Dialogue and Argumentation. An Examination of Douglas Walton's Theories of Reasoning, pp. 145-156. College Publications (2010)

18. Selvin, A.M., Buckingham Shum, S.: Hypermedia as a Productivity Tool for Doctoral Research. New Review of Hypermedia and Multimedia 11(1), 91-101 (2005)

19. Karacapilidis, N., Tzagarakis, M., Karousos, N., Gkotsis, G., Kallistros, V., Christodoulou, S., et al.: Tackling cognitively-complex collaboration with CoPe_it! International Journal of Web-Based Learning and Teaching Technologies 4(3), 22-38 (2009)

20. Debategraph. Debategraph: the global debate map (2011), http: / / debategraph.org/home (retrieved March 15, 2011)

21. Klein, M., Iandoli, L.: Supporting Collaborative Deliberation Using a Large-Scale Argumentation System: The MIT Collaboratorium. In: Proceedings of the Eleventh Directions and Implications of Advanced Computing Symposium and the Third International Conference on Online Deliberation (DIAC 2008/OD 2008), Berkeley, California, pp. 5-12 (2008)

22. Schneider, D., Voigt, C., Betz, G.: Argunet : A software tool for collaborative argumentation analysis and research. Paper presented at the 7th Workshop on Computational Models of Natural Argument (CMNA 2007), Hyderabad, India (2007)

23. van Gelder, T.: The rationale for Rationale. Law, Probability and Risk 6(1-4), 23-42 (2007)

24. ESTRELLA Project: Deliverable 4.1 - The Legal Knowledge Interchange Format (LKIF) (2008),

http://carneades.berlios.de/files/LKIF-Specification.pdf 